\title{
Una causa poco frecuente de muerte súbita e inesperada: dilatación gástrica aguda
}

\author{
Acute gastric dilatation: a rare case of sudden and unexpected \\ death
}

\author{
V. Ramos Medina ${ }^{1}$ \\ AM. López Calvo² \\ I. Palomo Gómez ${ }^{2}$ \\ J. Larrondo Espinosa ${ }^{3}$ \\ JL. Palomo Rando ${ }^{4}$
}

${ }^{1}$ Médico Forense, Jefe de Sección de

Histopatología.

${ }^{2}$ Médico Forense, Servicio de Patología, Instituto de Medicina

Legal, Málaga

${ }^{3}$ Facultativo del

Servicio de

Histopatología, Instituto Nacional de

Toxicología y Ciencias

Forenses, Sevilla

${ }^{4}$ Médico Forense, Jefe de Servicio de

Patología Forense.

Correspondencia:

V. Ramos Medina

Instituto de Medicina Legal,

Ciudad de la Justicia

Fiscal Luis Portero, 6 29010 Málaga

E-mail:valentin.ramos.ius@ juntadeandalucia.es

Fecha de recepción:

27. JUN. 2013

Fecha de aceptación

12. AGO. 2013

\section{Resumen}

Se presenta un caso de muerte súbita e inesperada en una mujer joven con antecedentes de esclerosis múltiple y espondilitis anquilosante que es encontrada muerta tras un breve cuadro de estreñimiento, dolor y distensión abdominal. En la autopsia, la apertura del abdomen mostró una extraordinaria dilatación del estómago repleto de secreciones. Este cuadro es un trastorno poco frecuente, de causa y patogenia desconocidas, que se relaciona con numerosas situaciones clínicas. El trastorno tiene una considerable mortalidad y un cuadro morfológico patognomónico en la autopsia.

Palabras clave: Dilatación gástrica. Muerte súbita.

\section{Abstract}

We report a case of sudden and unexpected death in a young woman with a history of multiple sclerosis and ankylosing spondylitis who was found dead after constipation, abdominal pain and bloating. At the autopsy the opening of the abdomen showed a largely distended stomach full of secretions. This condition is a rare disorder of unknown cause and pathogenesis, it is related to many clinical situations. The disorder has considerable mortality and the finding in the autopsy table is pathognomonic.

Key words: Gastric dilatation. Sudden death

\section{Introducción}

La dilatación aguda del estómago es un cuadro de escasa incidencia que se caracteriza por su potencial gravedad, con notable mortalidad, así como por su etiología y patogenia poco conocidas ${ }^{1}$. Se ha relacionado con postoperatorios de cirugía abdominal y pelviana con anestesia general, después de vagotomías y con varias situaciones patológicas como traumatismos contusos, quemaduras extensas, lesiones raquimedulares, corsé de yeso (cast syndro$m e)$, intoxicaciones por fenotiazinas y antidepresivos, diabéticos y sujetos con trastornos de la conducta alimentaria (anorexia nerviosa, bulimia nerviosa, trastorno por atracones, síndrome de Prader-Willi) y malnutridos que inician la nutrición por vía oral, entre otras ${ }^{1-3}$.
El cuadro de dilatación gástrica aguda guarda relación con el conocido como síndrome de Wilkie o síndrome de la arteria mesentérica superior. Éste es también un trastorno poco frecuente y se atribuye al pinzamiento de la tercera porción duodenal por la arteria mesentérica superior, descrito por primera vez por Rokitansky en $1842^{4}$. Es especialmente frecuente en sujetos muy delgados, en quienes la desaparición de la grasa intraabdominal hace que el ángulo aórtico-mesentérico se haga más agudo y se produzca una especie de pinza aórtico-mesentérica sobre el duodeno ${ }^{5}$. No obstante, este síndrome se describe como una afección distinta en algunos tratados ${ }^{1}$ y suele causar dilatación gastroduodenal de una forma menos violenta que la genuina dilatación gástrica ${ }^{6}$.

Hay muy pocos casos descritos en la literatura médica forense de dilatación gástrica aguda como 
causa de muerte súbita e inesperada7, algunos de ellos con perforación del órgano y la consiguiente peritonitis. Esto puede obedecer, además de a su escasa incidencia, a que la mayoría de las muertes por esta causa se producen en hospitales y no suelen requerir intervención forense. Por otra parte, el empleo sistemático de aspiración nasogástrica en el postoperatorio inmediato de la cirugía abdominal ha hecho disminuir aún más el número de casos.

\section{Presentación del caso}

Mujer de 38 años de edad diagnosticada de esclerosis múltiple en tratamiento con interferón beta y espondilitis anquilosante tratada con ibuprofeno y metamizol. El familiar que convivía con ella refiere que llevaba tres días sin defecar, con dolor y distensión abdominal. Sobre las 01:30 horas se administró un enema de $240 \mathrm{ml}$ (de los denominados laxantes por vía rectal) y se acostó. El referido familiar la encuentra a las 05 horas inconsciente y con restos de vómito verde oscuro en el rostro y en su entorno. Asistió el servicio médico de emergencias, que la encontró con signos de muerte cierta y no aplicó medidas de reanimación.

Hallazgos de la autopsia: talla,1,54 m; peso: 52 $\mathrm{kg}$; índice de masa corporal, normal $\left(21,9 \mathrm{~kg} / \mathrm{m}^{2}\right)$; restos de líquido verde oscuro en orificios nasales, en el interior y alrededor de la boca; distensión abdominal; al abrir el abdomen, gran dilatación del estómago y el duodeno que ocupa la mayor parte de la cavidad abdominal visible, Ilegando hasta la pelvis menor (Figura 1); las restantes vísceras abdominales quedan ocultas por el estómago (Figura 2), el cual se encuentra totalmente repleto de líquido verde oscuro (bilis y escasos restos hemáticos en forma de "posos de café"); las paredes del estómago están adelgazadas y con áreas de aspecto hemorrágico en la mucosa (Figuras 3 y 4); el estomago contiene 3,5 litros de líquido de coloración verde oscura; contenido gástrico en la vía aérea.

El estudio histopatológico diagnosticó, en el estómago, congestión en los vasos de la lámina propia y autólisis del epitelio glandular (Figura 5); en el encéfalo, placas inactivas de esclerosis múltiple (Figura 6); y además aspiración broncoalveolar.

El cribado toxicológico sólo detectó concentraciones subterapéuticas de metoclopramida, ibuprofeno y diazepam.

\section{Discusión}

La apertura del abdomen en los casos de muerte por esta causa muestra una imagen sorprendente e inconfundible: el estómago ocupa toda la cavidad

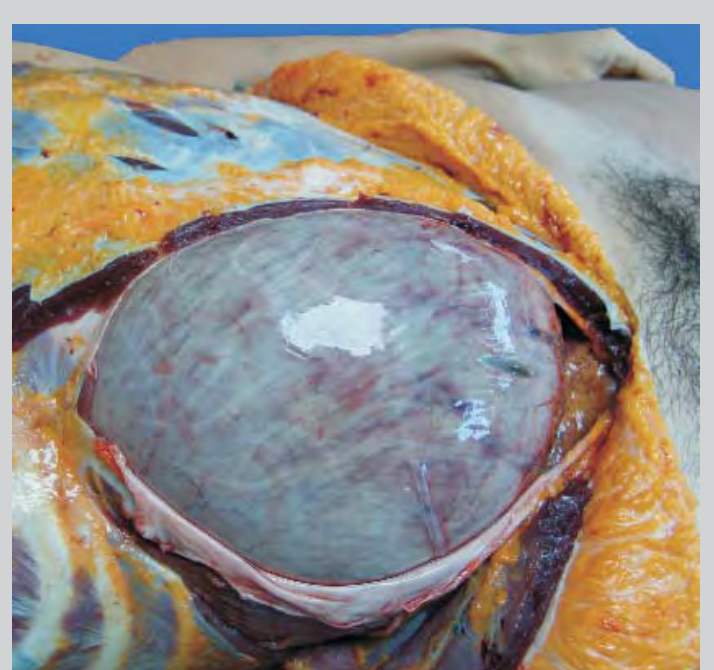

Figura 1.

Gran dilatación del estómago y duodeno que ocupa la mayor parte de la cavidad abdominal llegando hasta la pelvis menor.

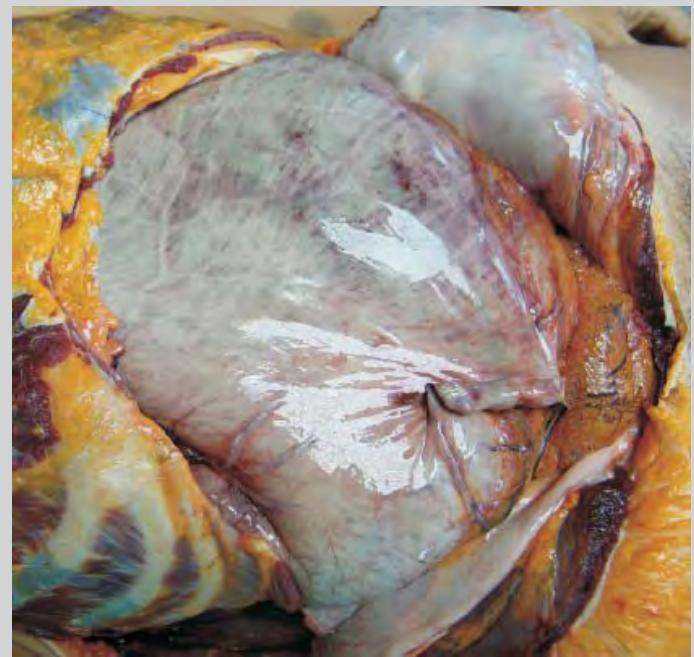

Figura 2.

Las restantes vísceras abdominales quedan ocultas por el estómago. 
Figura 3.

Tras revisión de tramo duodenal, el estómago es extraído aisladamente en primer lugar para evitar su rotura.

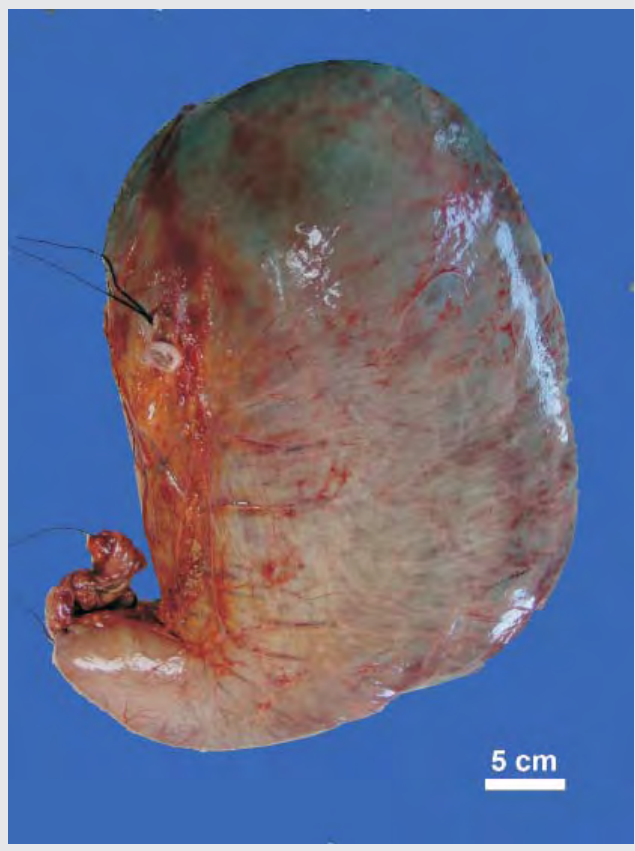

Figura 4. Paredes del estómago adelgazadas y con áreas de aspecto hemorrágico en la mucosa.

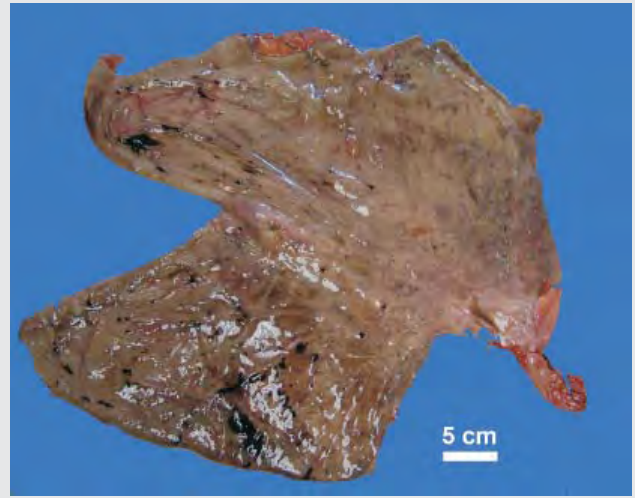

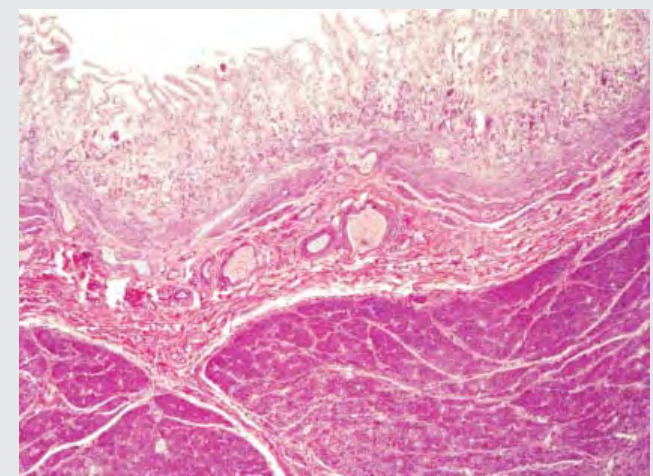

\section{Figura 5.}

Estómago. Autólisis del epitelio glandular con cierta congestión vascular submucosa (HE 2,5×).

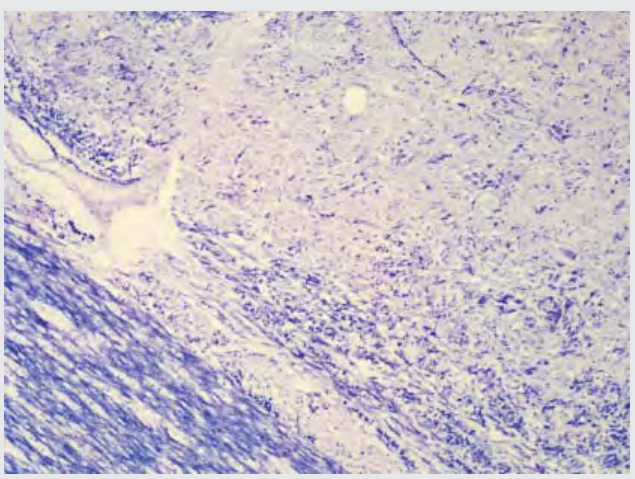

Figura 6.

Esclerosis múltiple. Detalle de la transición entre la zona mielinizada y la placa de esclerosis (técnica de Kluver Barrera; 10×). abdominal visible, llegando a la pelvis menor. En nuestro caso,el contenido gástrico (3,5 I de líquido) supone la pérdida de volumen de líquido circulante por acumulación en un tercer espacio; además, la compresión que ejerce el estómago dilatado sobre el área esplácnica y sobre la vena cava inferior disminuye el retorno venoso al corazón, y por ende es otro factor que favorece la aparición de choque hipovolémico como mecanismo que conduce a la muerte.

En cuanto a la causa, en el presente caso no había antecedentes de intervención quirúrgica ni trastorno de la conducta alimentaria, así que podría relacionarse con la esclerosis múltiple, habida cuenta de los casos reconocidos tras una lesión neurológica (lesiones medulares, vagotomías, neuropatía diabética ${ }^{8}$, etc.), y que dicha afección puede implicar a cualquier parte del sistema nervioso. Sin embargo, no hemos encontrado descrita esta complicación en la esclerosis múltiple.

\section{Conclusiones}

Hay dos factores que justifican la publicación de este caso: su excepcionalidad y la sorprendente e inconfundible imagen de la cavidad abdominal al proceder a su apertura en la autopsia. Incluso en pacientes 
sometidos a reanimación cardiopulmonar en quienes por error de técnica se intubó el esófago, la distensión del estómago no alcanza nunca el volumen de la dilatación aguda del estómago, ni por supuesto el extraordinario contenido de líquido acumulado en su interior. Si bien existen casos descritos de muerte por dilatación gástrica aguda, en general se asocian a perforación, necrosis o rotura de la pared del estómago.
Hay muy pocos casos sin rotura; en concreto, en la literatura médica sólo hemos encontrado otro $\mathrm{Caso}^{7}$.

Aunque es un cuadro infrecuente, conviene que sea conocido por los patólogos forenses.

Los autores declaran no tener conflicto de intereses.

\section{Bibliografía}

1. Méndez Martín J. Afecciones obstructivas del estómago y duodeno. En: Duran Sacristán H. Cirugía. Patología y clínicas quirúrgicas. $2^{a}$ ed. Madrid: McGraw-Hill-Interamericana de España; 1993. p. 2307-12.

2. Trullenque R. Cirurgia abdominal. Dilatació aguda gastrica. Valencia: Universitat de Valencia; 2002.

3. Schrander-Stumpel CT, Curfs LM, Sastrowijoto $P$, Cassidy SB, Schrander JJ, Fryns JP. Prader-Willi syndrome: causes of death in an international series of 27 cases. Am J Med Genet A. 2004;124A:3338.

4. Todd SR, Marshall GT, Tyroch AH. Acute gastric dilatation revisited. Am Surg. 2000;66:709-10.
5. Bauer S, Karplus R, Belsky V, Mha HA. Superior mesenteric artery syndrome: a forgotten entity. Isr Med Assoc J.2013;15:189-91.

6. Kennedy KV, Yela R, Achalandabaso MM, MartínPérez E. Superior mesenteric artery syndrome: diagnostic and therapeutic considerations. Rev Esp Enferm Dig. 2013;105:236-8.

7. Watanabe S, Terazawa K, Asari M, Matsubara K, Shiono H, Shimizu K. An autopsy case of sudden death due to acute gastric dilatation without rupture. Forensic Sci Int. 2008;180:e6-e10.

8. Featherstone PJ, George LD. Gastric dilatation secondary to diabetic autonomic neuropathy. N Eng/ J Med. 2005;353:25. 